\title{
Utility of free/total prostate specific antigen (f/t PSA) ratio in diagnosis of prostate carcinoma
}

\author{
V. Thakur ${ }^{\mathrm{a}, *}$, P.P. Singh ${ }^{\mathrm{b}}$, M. Talwar ${ }^{\mathrm{c}}$ and U. Mukherjee ${ }^{\mathrm{d}}$ \\ ${ }^{a}$ Biochemistry Division, Department Of Laboratory Medicine, Batra Hospital \& Medical Research Centre, New \\ Delhi, India, 110062 \\ ${ }^{\mathrm{b}}$ Department Of Urosurgery, Batra Hospital \& Medical Research Centre, New Delhi, India, 110062 \\ ${ }^{\mathrm{c}}$ Department Of Urosurgery, Batra Hospital \& Medical Research Centre, New Delhi, India, 110062 \\ ${ }^{d}$ Pathology Division, Department Of Laboratory Medicine, Batra Hospital \& Medical Research Centre, New Delhi, \\ India, 110062
}

\begin{abstract}
The discovery that PSA exists in serum in both free and complexed forms led to development of immunoassays specific for different PSA forms. This helped in measuring free PSA in the presence of PSA-ACT (PSA- $\alpha$ antichymotrypsin), hence it was possible to calculate the percent free PSA or free to total PSA ratio, measurement of which was helpful in reducing the number of unnecessary biopsies significantly, while maintaining a high clinical sensitivity for detection of cancer. The study was performed on 103 consecutive male patients (mean age $68 \pm 10.8$ years SD) comprising of 90 patients with benign disease $(87 \%)$ and 13 prostate carcinoma patients $(13 \%)$, who had histologically proven prostate cancer. Patients with total PSA between $2-25 \mathrm{ng} / \mathrm{ml}$ were included in the study. 30 normal healthy males with age $58 \pm 10$ years, served as control. Serum total PSA and free PSA were analyzed using streptavidin biotin EIA method (M/s Roche Diagnostics, Germany). The mean total PSA in normal healthy control subjects was $1.86 \pm 1.07 \mathrm{ng} / \mathrm{ml}$. It was increased significantly in diseased condition. Its mean concentration in carcinoma patients was $12.6 \pm 5.3 \mathrm{ng} / \mathrm{ml}$ and in benign patients it was $6.3 \pm 4.6 \mathrm{ng} / \mathrm{ml}$. The free to total PSA ratio in all the three groups was significantly different $(p<0.004)$ from each other. In carcinoma patients, mean f/t PSA ratio was $0.12 \pm 0.06$ as compared to $0.21 \pm 0.11$ and $0.28 \pm 0.17$ in benign patients and in control respectively. The sensitivity and specificity of the test was calculated at different $\mathrm{f} / \mathrm{t}$ PSA ratio cutoff. At 0.1 cutoff value, sensitivity of the test was $54 \%$ and specificity was $83 \%$. The positive predictive value (ppv) was $32 \%$ and negative predictive value (npv) was $92 \%$. From cutoff value of 0.12 to 0.16 , sensitivity was increased from $54 \%$ to $85 \%$ but specificity was reduced from $78 \%$ to $67 \%$.The ppv did not show much change and npv was increased from $92 \%$ to $97 \%$. Increasing the cut off value thereafter showed no change in sensitivity but specificity was further reduced to $40 \%$, therefore in this patient series, f/t PSA ratio cutoff of 0.16 was found to be the appropriate cutoff value. Combination of this ratio cutoff with other parameters like serum total PSA, DRE and TRUS helped in increasing the sensitivity of the test and this also helped in reducing the number of unnecessary biopsies. In 103 men who were biopsied, 13 (12.6\%) prostatic carcinoma were identified. Among these 13 cancer patients, 9 patients had abnormal findings in DRE.7 individuals out of these 9, also had free to total PSA ratio lower than 0.16 and would have been biopsied and diagnosed anyway. If we use only f/t PSA ratio less than 0.16 , to decide whom to biopsy, we would have biopsied and diagnosed 11/13 cases i.e. sensitivity of $85 \%$ but If we decide to biopsy those patients who had abnormal DRE and those who had low f/t PSA ratio, we could identify 13/13 carcinoma i.e. $100 \%$ sensitivity.

Combining the f/t PSA ratio with total PSA, DRE and TRUS findings could help in reducing the number of unnecessary biopsies. 37 patients who were negative for malignancy having total PSA in the range of 5-20 ng/ml, normal DRE and TRUS findings, have been biopsied but with combination of total PSA in the range of 5-20 ng/ml, normal findings in digital rectal examination and TRUS and f/t PSA ratio more than 0.16 (cutoff), we could have avoided 16 biopsies which were unnecessary that means there was $43 \%$ reduction in unnecessary biopsies.
\end{abstract}

* Corresponding author: Dr. Vinita Thakur, Sr. Biochemist, Biochemistry Division, Department of Laboratory Medicine, Batra Hospital \& Medical Research centre, 1, Tughlakabad Institutional Area, MB Road, New Delhi 110062, India. Tel.: +91 11 22448083; Fax: +91 11 29957661; E-mail: vinitathakur@hotmail.com.

\section{Introduction}

Benign prostatic hyperplasia and prostate cancer, are the two most common forms of prostatic disease found in men beyond the age of 50 years. Although the age 
of onset for both of these diseases is same, they are to be clinically differentiated in early stages for treatment to be most effective [1]. In this endeavor, prostate specific antigen (PSA) has become a key tumor marker for screening and early detection of prostate cancer [2]. Although prostate specific antigen can be used for effective diagnosis of clinically significant cancer, its use is limited by a number of benign conditions like benign prostatic hyperplasia, prostatitis, aging, prostatic infarction etc. that can cause elevations in serum total PSA. The lack of greater specificity of total PSA testing has lead in an increase in number of unnecessary biopsies for clinically detecting carcinoma in patients having elevated serum total PSA [3]. In recent efforts to increase the specificity of total PSA testing, knowledge of PSA in different molecular forms has proven to be of great utility. Prostate specific antigen in serum occurs in different molecular forms and the concentration of these molecular forms may vary according to the disease state of the prostate gland [4]. PSA complexes with serine protease inhibitors, most notably $\alpha-1$ - antichymotrypsin and $\alpha_{2}$-macroglobulin. The measurement of total serum PSA concentration represents the sum of the uncomplexed PSA or free PSA and the PSA complexed with $\alpha_{1}$-antichymotrypsin 5 . It is known that proportion of complexed PSA is higher in men with prostate cancer than in men with benign disease hence it is suggested that a better differentiation between benign disease and prostate cancer is accomplished by calculating the ratio of complexed PSA to total PSA or the ratio of free PSA to total PSA [6,7]. Although PSA$\mathrm{ACT}$ is the predominant serum PSA form measured by immunoassay [8], the PSA- ACT immunoassay using anti PSA monoclonal antibodies for capture and antiACT monoclonal antibodies for detection may be compromised by interference of serum cathepsin $\mathrm{G}(\mathrm{cG})$ ACT complex [9]. However, this improved immunoassay has shown minimal improvement in prostate cancer detection as compared to ratio of free PSA to total PSA or percent free PSA. According to Catalona et al., the ratio of free PSA to PSA ACT was also not superior to percent free PSA in improving cancer detection specificity [9].

The present study is planned to evaluate the utility of free to total PSA ratio in discriminating cancer from benign disease in men who presented with prostatic symptoms and had total PSA in the range of $2-25 \mathrm{ng} / \mathrm{ml}$.

\section{Materials and methods}

The study was performed on 103 consecutive male patients (mean age $68 \pm 10.8$ years SD) compris- ing of 90 patients with benign disease $(87 \%)$ and 13 prostate carcinoma patients $(13 \%)$ who had histologically proven prostate cancer. The diagnosis of benign disease was established clinically by digital rectal examination, DRE (enlarged, confined, indolent prostate with smooth surface and no induration) and transrectal ultrasound, TRUS (enlarged prostate without hypoechoic area or asymmetry suspicious for prostate carcinoma). The malignancy in these patients was excluded by concomitant trucut biopsy. The biopsy was taken using springloaded trucut biopsy needle(Microvasive Boston Scientific). 8 to 12 cores of the biopsy were taken in all cases subject to size of the gland. All carcinoma patients were diagnosed clinically, radiologically and disease was proved histologically by trucut needle biopsy of posterior zone with transurethral resection of prostate including transitional and central zones. Those cases presenting with LUTS in whom no clinical or radiological evidence of prostatic enlargement was found were not included in the study. Thirty normal healthy males with mean age $58 \pm 10$ years served as control.

To avoid possible errors caused by the release of PSA from the prostate gland on manipulative procedures, blood samples were drawn from these patients before any prostate manipulation. The samples were processed immediately or stored at $-20^{\circ} \mathrm{c}$ for next day processing.

Serum total PSA and free PSA were analyzed using streptavidin biotin EIA method (M/s Roche Diagnostics, Germany). The monoclonal antibodies used in the estimation of total PSA were highly specific for the free PSA and $\alpha$-1- antichymotrypsin complex and the monoclonal antibodies used in the analysis of fPSA was highly specific for fPSA molecule.

For total PSA analysis, $50 \mu$ ls of sample was incubated with $1000 \mu \mathrm{ls}$ of incubating buffer in streptavidin tubes for 90 minutes. Incubating buffer contains phosphate buffer with biotinylated monoclonal anti PSA antibodies and peroxidase labeled monoclonal anti PSA antibodies. Solution was discarded and tubes were washed vigorously thrice with washing buffer. $1000 \mu$ ls Chromogen (di-ammonium 2, 2' - azinobis (3ethyl benzothiazoline- 6- sulphonate) was added. After 30 min of incubation, absorbance was read at $405 \mathrm{~nm}$ wavelength. Concentration of serum total PSA was calculated.

For FPSA analysis, $70 \mu \mathrm{ls}$ of sample was incubated for 30 minutes with $1000 \mu \mathrm{ls}$ of incubation buffer in streptavidin tubes. The incubation solution contains biotinylated anti PSA antibodies in phosphate buffer. After first incubation, tubes were washed vigorously 
with washing buffer for three to four times. Second incubation was done for 30 minutes with conjugate buffer containing monoclonal anti PSA antibodiesperoxidase conjugate. Again tubes were washed thrice with washing buffer and then incubated with chromogen for 30 minutes. Absorbance was read at $405 \mathrm{~nm}$ and concentration of free PSA was determined.

$\mathrm{f} / \mathrm{t}$ PSA ratio was calculated using these values.

\subsection{Statistical analysis}

Concentration of total PSA, free PSA and their ratio was expressed as mean $\pm \mathrm{SD}$. One way analysis of variance (ANOVA) was applied to calculate the $p$ value. Sensitivity and specificity of test was calculated at different $\mathrm{f} / \mathrm{t}$ PSA ratio cutoff values.

\section{Results}

Patients with total PSA between 2-25 ng/ml were included in the study. The mean total PSA in control population was $1.86 \pm 1.07 \mathrm{ng} / \mathrm{ml}$. It was increased significantly in diseased condition. Its mean concentration in carcinoma prostate was $12.6 \pm 5.3 \mathrm{ng} / \mathrm{ml}$ and in patients with benign disease, it was $6.3 \pm 4.67 \mathrm{ng} / \mathrm{ml}$. The mean concentrations in all the three groups were significantly different from each other $(p<0.000)$. The mean free PSA concentration in control subjects was $0.40 \pm 0.25 \mathrm{ng} / \mathrm{ml}$. In carcinoma patients, it was $1.5 \pm 1.0 \mathrm{ng} / \mathrm{ml}$ and in benign patients, it was $1.15 \pm$ $0.9 \mathrm{ng} / \mathrm{ml}$. The difference in mean free PSA concentration was nonsignificant $(p<0.14)$ However, its concentration was significantly higher in both these groups as compared to control $(p<0.05)$. The free to total PSA ratio in all the three groups was significantly different $(p<0.004)$ from each other. In carcinoma patients, mean f/t PSA ratio was $0.12 \pm 0.06$ as compared to $0.21 \pm 0.11$ and $0.28 \pm 0.17$ in patients with benign disease and in control respectively (Table 1).

The sensitivity and specificity of the f/t PSA ratio was calculated at different cut off values (Table 2). At 0.1 cutoff value, sensitivity of the test was $54 \%$ and specificity was $83 \%$. The positive predictive value (ppv) was $32 \%$ and negative predictive value (npv) was $92 \%$. From cutoff value of 0.12 to 0.16 , sensitivity increased from $54 \%$ to $85 \%$ but specificity reduced from $78 \%$ to $67 \%$. The ppv did not show much change and npv was increased from $92 \%$ to $97 \%$. Increasing the cut off value thereafter showed no change in sensitivity but specificity was further reduced to $40 \%$, hence in our patient series f/t PSA ratio cutoff of 0.16 was found to be appropriate cutoff value.

Combination of the $f / t$ PSA ratio with other clinical and biochemical parameters i.e. DRE, TRUS findings and serum total PSA concentration helped in increasing the sensitivity of the test and could also reduce the number of unnecessary biopsies performed. In 103 men who were biopsied, $13(12.6 \%)$ prostatic carcinoma cases were identified. Among these 13 cancer patients, 9 had abnormal findings in DRE. Out of them, 7 individuals also had f/t PSA ratio lower than 0.16 and would have been biopsied and diagnosed anyway. If we use only f/t PSA ratio lower than 0.16 to decide whom to biopsy, we would have biopsied and diagnosed 11/13 cases i.e. sensitivity of $85 \%$ but If we decide to biopsy those patients who had abnormal findings in DRE and those who had low f/t PSA ratio we could identify 13/13 carcinoma i.e. $100 \%$ sensitivity. Combining the f/t PSA ratio with total PSA, DRE and TRUS findings could help in reducing the number of unnecessary biopsies. 37 patients who were negative for malignancy, having total PSA in the range of 5-20 ng/ml and normal DRE and TRUS have been biopsied. Out of these 37 patients, 16 patients had f/t PSA ratio more than 0.16 and 21 patients had $\mathrm{f} / \mathrm{t}$ PSA ratio less than 0.16 . Combination of total PSA in the range of 5-20 ng/ml, normal findings in digital rectal examination, TRUS and f/t PSA ratio more than 0.16 could have avoided 16 biopsies which were unnecessary that means there was $43 \%$ reduction in unnecessary biopsies performed.

\section{Discussion}

Although total serum PSA concentration performs poorly in differentiating histologic BPH and organ confined prostate cancer, serum PSA measurements have been utilized for prostate cancer screening $[9,10]$. However, in such screening studies only a select subset of men are subjected to a transrectal ultrasound (TRUS) guided biopsy, while the remainder of the population does not undergo a biopsy despite possibly harboring undiagnosed prostate cancer, this leads to overestimation in the number of false negative cases.

Therefore, there is a pressing need of another tumor marker which can be used in differentiating benign disease from prostate carcinoma in conjunction with digital rectal examination, TRUS and high PSA.

The discovery that the serum PSA exists in both free and complexed (PSA- $\alpha$ antichymotrypsin) forms led to resurgence in research on the biochemistry of PSA 
Table 1

Total PSA, Free PSA and f/t PSA ratio in prostatic patients as well as control

\begin{tabular}{lccc}
\hline Parameters & Carcinoma Prostate & Benign Disease & Control \\
\hline $\begin{array}{l}\text { Total No. of Patients } \\
\text { Total PSA (ng/ml)* }\end{array}$ & 13 & 90 & 30 \\
$($ Mean \pm SD) & $12.6 \pm 5.3$ & $6.3 \pm 4.67$ & $1.86 \pm 1.07$ \\
$\begin{array}{l}\text { Free PSA(ng/ml) } \\
(\text { Mean } \pm \text { SD) }\end{array}$ & $11.5 \pm 1.0$ & $21.15 \pm 0.9$ & $30.40 \pm 0.25$ \\
f/t PSA ratio*** & $0.12 \pm 0.06$ & $0.21 \pm 0.11$ & $0.28 \pm 0.17$ \\
\hline
\end{tabular}

$p$ value ${ }^{*}<0.000$

${ }^{* * 1} \mathrm{vs}^{2}<0.14,{ }^{1,2} \mathrm{vs}^{3}<0.05$.

$* * *<0.004$.

Table 2

The sensitivity and specificity of the f/t PSA ratio calculated at different cut off values. The patients having Total PSA in the range of $2-25 \mathrm{ng} / \mathrm{ml}$ were included in the study

\begin{tabular}{lcccccccccc}
\hline f/t PSA ratio cutoff values & 0.10 & 0.12 & 0.13 & 0.14 & 0.15 & 0.16 & 0.17 & 0.18 & 0.20 & 0.22 \\
\hline Sensitivity (\%) & 54 & 54 & 54 & 54 & 69 & 85 & 85 & 85 & 85 & 85 \\
Specificity (\%) & 83 & 78 & 75 & 71 & 70 & 67 & 63 & 54 & 51 & 40 \\
1-specificity (\%) & 17 & 22 & 25 & 29 & 30 & 33 & 37 & 46 & 49 & 60 \\
Positive predictive value (\%) & 32 & 26 & 24 & 21.2 & 25 & 26.8 & 25 & 21 & 20 & 17 \\
Negative predictive value (\%) & 92 & 92 & 92 & 91.4 & 94 & 97 & 97 & 96 & 96 & 95 \\
\hline
\end{tabular}

and development of immunoassays specific for different PSA forms [11,12]. The development of these immunoassays helped in measuring free PSA in the presence of PSA- $\alpha$ antichymotrypsin (PSA-ACT), hence it was possible to calculate the percent free PSA or free to total PSA ratio, which could be utilized to reduce the number of unnecessary biopsies significantly, while maintaining a high clinical sensitivity for detection of cancer $[13,14]$.

In present study, we have selected a subset of patients who had total PSA in the range of $2-25 \mathrm{ng} / \mathrm{ml}$. There are many studies available mentioning the utility of $f / t$ PSA ratio in patients having total PSA as low as $1.8 \mathrm{ng} / \mathrm{ml}$ and as high as $25 \mathrm{ng} / \mathrm{ml}$ i.e. outside the range of total PSA between 4 to $10 \mathrm{ng} / \mathrm{ml}$ (the gray zone). Catalona et al. [10] have documented in one of their multiinstitutional trial of over 700 men that a significant improvement in cancer detection was found when percent free PSA or free PSA ratio is being used as predictor. The sensitivity of the test was greater than $95 \%$ and this was found among men who presented with serum total PSA between 4-10 ng/ml. In another study in a significant number of prostatic patients $(n=308)$, Van Cangh et al. had suggested that the routine use of free to total PSA might be advantageous in discriminating cancer and benign hyperplasia in the diagnostic zone of $1.8-10 \mathrm{ng} / \mathrm{ml}$ of total PSA [11]. Morgan et al. have proposed the use of $f$ PSA to total PSA ratio cut off of 0.1 which maintained the sensitivity of $91 \%$ with corresponding specificity of $86 \%$. The cut off range for total PSA was $4.1-24.8 \mathrm{ng} / \mathrm{ml}$ in their patient population [12].
In our patient series the total PSA in carcinoma patients was significantly higher than benign cases as well as control. Mean free PSA concentration in carcinoma prostate patients and benign patients was not significantly different from each other, resulting in low $\mathrm{f} / \mathrm{t}$ PSA ratio in carcinoma as compared to benign patients. This observation suggests that the cancer prostate predominantly produce complexed PSA. Lilja had reported that prostate cancer produces predominantly complexed PSA resulting in low f/t PSA ratio [16]. This behavior of PSA molecular forms can be used as a good diagnostic tool for differentiation of prostate cancer and noncancer .Determination of molecular forms of PSA has been shown to significantly enhance prostate cancer detection [15]. Discrimination between prostate cancer and benign disease is more problematic in patients with intermediate total prostate specific antigen values $4-12 \mathrm{ng} / \mathrm{ml}$. Such patients require invasive procedures to be performed to confirm the malignancy. In such cases, to avoid unnecessary biopsies, noninvasive method like estimation of free to total PSA ratio and its use in conjunction with total PSA more than $5 \mathrm{ng} / \mathrm{ml}$ and digital rectal examination have been developed [16]. According to Ali Vizatos et al. [17] in their patient series, the total PSA did not vary significantly between benign and malignant conditions, However the $\mathrm{f} / \mathrm{t}$ PSA ratio was significantly lower among the carcinoma prostate patients $(p<0.001)$.

The use of f/t PSA ratio has also helped in increasing the sensitivity of the test. In our patient series, sensitivity of the test was $54 \%$ with specificity of $83 \%$ 
at $\mathrm{f} / \mathrm{t}$ PSA cutoff of 0.1 . At 0.16 cutoff, sensitivity of the test was increased to $85 \%$ but specificity dropped to $67 \%$. Increasing the $\mathrm{f} / \mathrm{t}$ PSA ratio thereafter did not show any change in sensitivity but specificity dropped further to $40 \%$. Luderer et al. have reported a sensitivity of $80 \%$ and a specificity of $49 \%$ at a cut off point of $0.2 \mathrm{f} / \mathrm{t}$ PSA with respect to the differentiation of men with prostate cancer and BPH as determined by negative TRUS biopsy. No patient with prostate cancer had a f/t PSA ratio of more than 0.25 or $25 \%(100 \%$ sensitivity) [18]. Oesterling et al. have recommended a cutoff of 0.15 for the $\mathrm{f} / \mathrm{t}$ PSA ratio as upper limit for diagnosis of prostate cancer [19].

Apart from increasing the sensitivity of the test, it also helped in reducing the number of unnecessary biopsies. In our patient series, combination of total PSA more than $5 \mathrm{ng} / \mathrm{ml}$ and normal DRE and free to total PSA ratio. More than 0.16 , helped in reducing performance of unnecessary biopsies to one half. If we combine total PSA more than $5 \mathrm{ng} / \mathrm{ml}$, f/t PSA ratio lower than 0.16 and normal DRE to confirm malignancy, out of 37 patients all negative for cancer, only 21 patients needed biopsy i.e. $43 \%$ reduction in biopsy performance. Alivizatos et al. have shown $48 \%$ reduction in number of biopsies when they decided to biopsy only those patients who had abnormal findings in DRE or TRUS and those who had low f/t PSA ratio [17].

\section{Conclusion}

we conclude that determination of $\mathrm{f} / \mathrm{t}$ PSA ratio in patients having serum total PSA concentration in the range of 2-20 ng/ml, combined with digital rectal examination and TRUS will help in increasing the sensitivity of the test and reduce the number of unnecessary biopsies in these patients.

\section{Acknowledgements}

We sincerely thank Batra Medical Research Center to permit us to carry out and compile this work in the Department of Laboratory Medicine and Urosurgery.

\section{References}

[1] W.J. Catalona, D.S. Smith, T.L. Ratliff and J.W. Roster, Detection of organ confined prostate cancer is increased through prostate specific antigen based screening, JAMA 270 (1993), 948-954.
[2] T.A. Stamey, N. Yang, A.R. Hay, J.E. Mcneal, F.S. Freiha and E. Redwine, Prostate specific antigen as a serum marker for adenocarcinoma of the prostate, N Eng J Med 317 (1987), 909-916.

[3] A.W. Partin, H.B. Carter, D.W. Chan, J.I. Epstein, J.E. Oesterling, R.C. Rock, J.P. Weber and P.C. Walsh, Prostate specific antigen in the staging of localized prostate cancer, influence of tumor differentiation, tumor volume and benign hyperplasia, J Urol 143 (1990), 747-752.

[4] K. Jung, B. Brux, M. Lein et al., Determination of alphaantichymotrypsin-PSA complex in serum does not improve the differentiation between benign prostatic hyperplasia and prostate cancer compared with total PSA and percent free PSA, Urology 53(6) (1999), 1160-1168.

[5] W.J. Catalona, Clinical utility of measurements of free and total prostate specific antigen (PSA): A review, Prostate 7 (1996), 64-69.

[6] P.J. Van Cangh, P. De Nayer, R. De V Ischer, P. Sanvage, B. Tombal, F. Lortge, F.X. Wese and R. Opsomer, Free to total prostate specific antigen (PSA) ratio improves the discrimination between prostate cancer and benign prostatic hyperplasia (BPH) in the diagnostic gray zone of 1.8 to $10 \mathrm{ng} / \mathrm{ml}$ total PSA, Urology 48(6A) (1996), 67-70.

[7] W.J. Catalona, A.W. Partin, J.A. Finlay, D.W. Chan, S.H.G. Rittenhouse, R.L. WolFert and D.L. Woodrum, Use of Percentage of free prostate specific antigen to identify men at high risk of prostate cancer when PSA levels are 2.51 to $4 \mathrm{ng} / \mathrm{ml}$, Urology 54(2) (1999), 220-224.

[8] T.O. Morgan, D.G. Mckod, E.J. Sleifer, G.P. Murphy and J.W. Moul, Prospective use of free prostate specific antigen to avoid repeat prostate biopsies in men with elevated total prostate specific antigen, Urology 48(6A) (1996), 76-80.

[9] W.J. Catalona, D.S. Smith, T.C. Ratliff, K.M. Dodds, D.E. Soplen, J.J.J. Ynan J.A. Petros and G.L. Andriole, Measurement of prostate specific antigen in serum as a screening test for prostate cancer, N. Engl J Med 324 (1991), 1156-1161.

[10] M.K. Brawer, M.P. Chetner, J. Beatle, D.M. Buchner, R.L. Vesselia and R.H. large, screening for prostatic carcinoma with prostate specific antigen, J Urol 147 (1992), 841-845.

[11] A. Christensson, C.B. Laurel and H. Lilja, Enzymatic activity of prostate specific antigen and its reactions with extracellular serine protease inhibitor, Eur J Biochem 194 (1990), 755-763.

[12] H. Lilja, A. Christensson, U. Dahlen, M.T. Matikainen, O. Nilsson, K. Petterson and T. Lovgen, Prostate specific antigen in serum occurs predominantly in complex with alpha 1-antichymotrypsin, Clin Chem 37 (1991), 1618-1625.

[13] G.M. Marley, M.C. Miller, M.W. Kattan, G. Zhao, K.P. Patton, R.L. Vessella, G.L. Wright, Jr, P.F. Schellhammer and R.W. Veltri, Free and complexed prostate specific antigen serum ratios to predict probability of primary prostate cancer and benign prostatic hyperplasia, Urology 48 (1996), 16-22.

[14] A. Reissigl, H. Klocker, J. Pointner, K. Fink, W. Horninger, O. Ennemoser, H. Strasser, K. Colleselli, L. Holti and G. Bartsch, Usefulness of the ratio free/total prostate specific antigen in addition to total PSA levels in prostate cancer screening, Urology 48 (1996), 62-66.

[15] W.J. Catalona, D.S. Smith, R.L. Wolfert, T.J. Wang, H.G. Rittenhouse, T.L. Ratliff and R.B. Nadler, Evaluation of percentage of free serum prostate specific antigen to improve specificity of prostate cancer screening, JAMA 274 (1995), 12141220 .

[16] H. Lilja, Significance of different molecular forms of serum PSA, the free noncomplexed form of PSA versus that com- 
plexed to alpha 1- antichymotrypsin, Urol. Clin. North. Ame. 20 (1993), 681-686.

[17] G. Alivizatos, C. Deliveliotis, D. Mitropoulos, G. Raptides, G. Louras, K.A. arayiannes, T. Becopoulos and A.M. Dimopoulos, Does free to total ratio of prostate specific antigen alter decision making on prostate biopsy? Urology 48(6A) (1996), 71-75.

[18] A.A. Luderer, Y.T. Chen, T.F. Soriano, W. Kramp, G. Carlson, C. Cuny, T. Sharp, W. Smith, Petteway, M. Brawer and R. Thiel, Measurement of the proportion of free to total prostate specific antigen improves diagnostic performance of prostate specific antigen in the diagnostic grey zone of total prostate specific antigen, Urology 46 (1994), 187-194.

[19] J.E. Oesterling, S.J. Jacobson, G.G. Klee, K. Peterson, T. Piironen, P.A. Abrahmsson, U.H. Stenman, B. Dowell, T. Lorgren and H. Lilja, Free complexed and total serum prostate specific antigen. The establishment of appropriate reference ranges for their concentrations and ratios, J Urol 159 (1995), 1090-1095. 


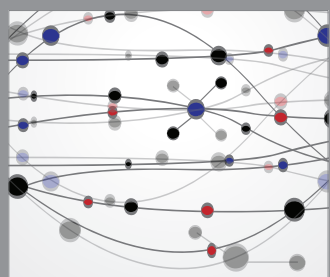

The Scientific World Journal
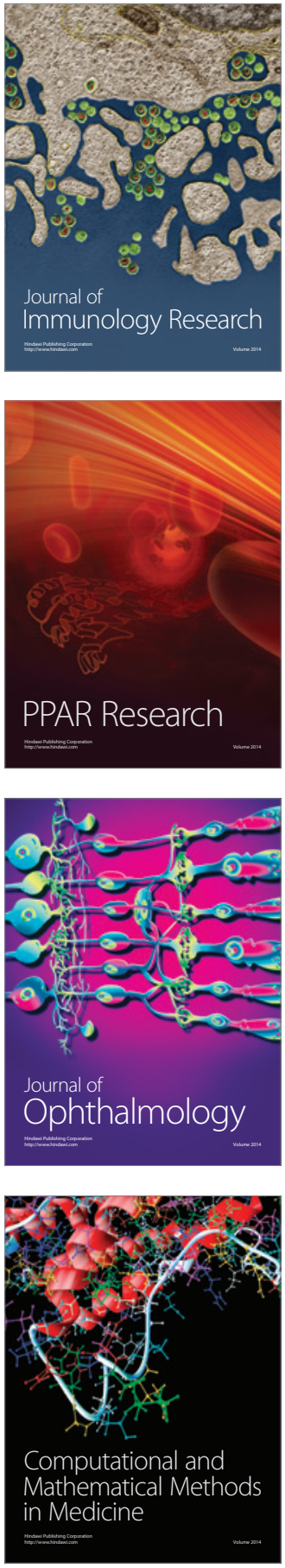

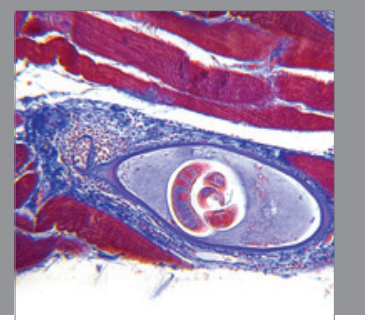

Gastroenterology

Research and Practice
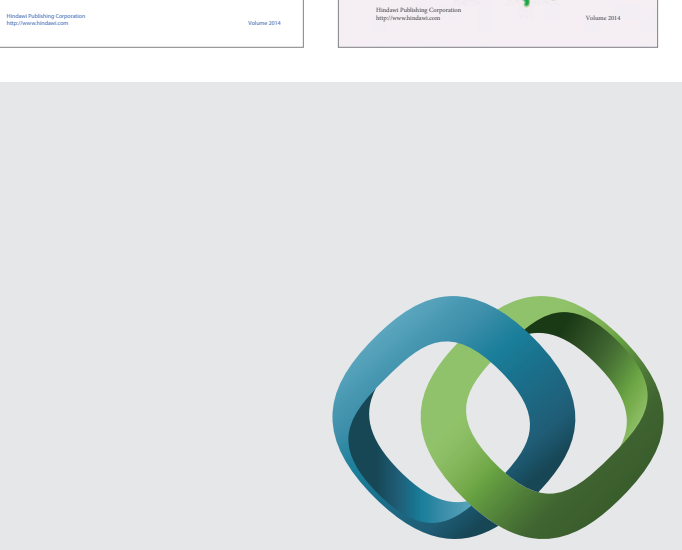

\section{Hindawi}

Submit your manuscripts at

http://www.hindawi.com
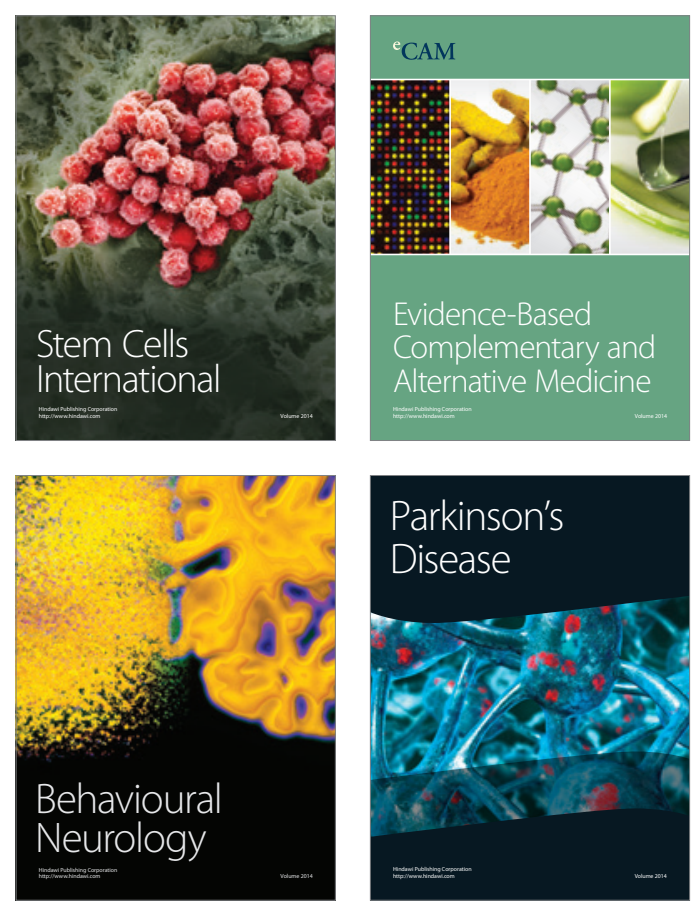

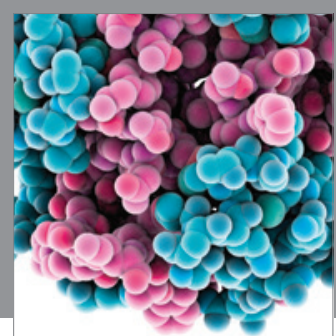

Journal of
Diabetes Research

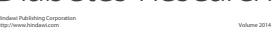

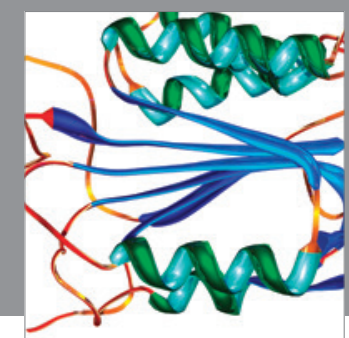

Disease Markers
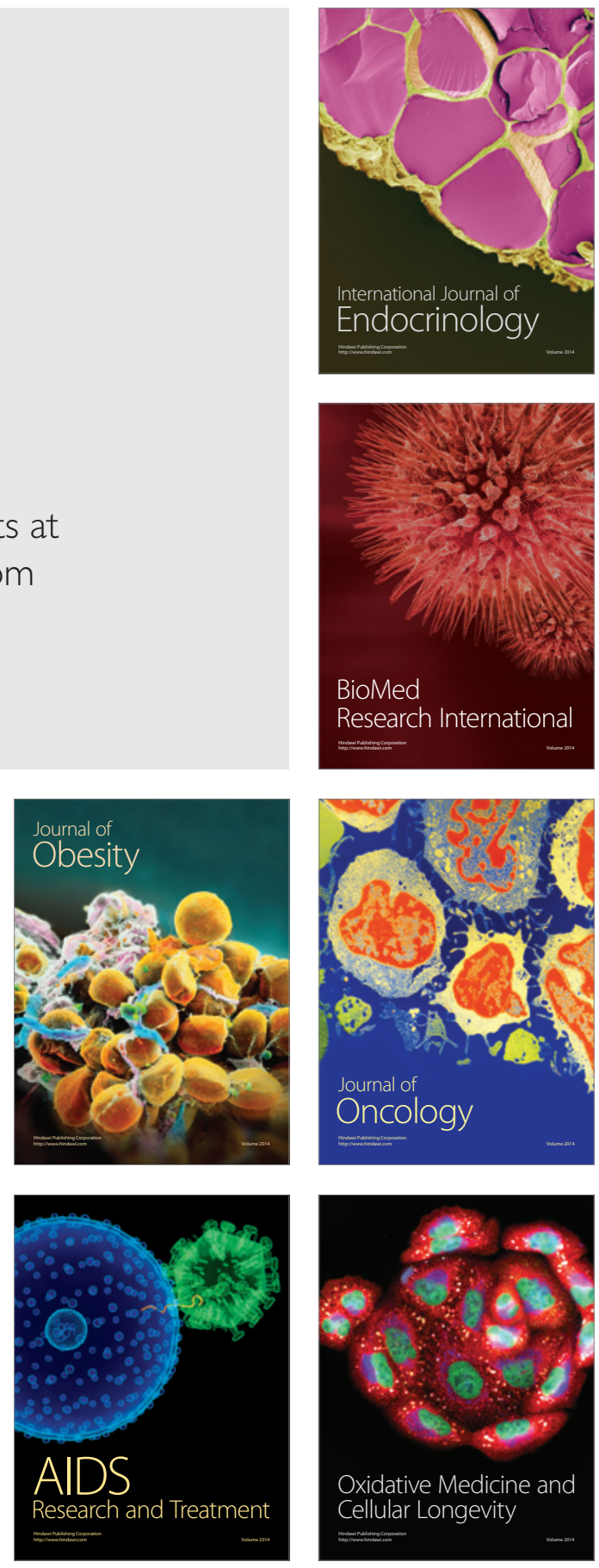